\title{
The effect of the introduction of the ERAS protocol in laparoscopic total mesorectal excision for rectal cancer
}

\author{
C. J. A. Huibers • M. A. J. de Roos • K. H. Ong
}

Accepted: 1 December 2011 / Published online: 16 December 2011

(C) The Author(s) 2011. This article is published with open access at Springerlink.com

\begin{abstract}
Objective The aim of the study was to determine whether the introduction of the Enhanced Recovery after Surgery (ERAS) protocol in laparoscopic total mesorectal excision (TME) for rectal cancer offers additional advantages concerning postoperative hospital stay compared to laparoscopy and conventional care.

Methods A consecutive series of patients that underwent a laparoscopic TME for rectal cancer in a single institution between January 2004 and July 2009 were retrospectively included in this study. The ERAS protocol was introduced in this cohort in January 2007. The study cohort was divided in a conventional care group and an ERAS group. Both groups were compared for primary and secondary outcome measures. The primary outcome measure was postoperative length of hospital stay.

Results Seventy-six patients were included: 43 in the ERAS group and 33 in the conventional care (control) group. Median hospital stay was 7 days (range 2-83 days) in the ERAS group and 10 days (range 4-74 days) in the control group $(p=0.04)$. Return of bowel function occurred on days 2 and 3 respectively $(p<0.001)$. There were no significant differences between both groups concerning postoperative complications, readmission rate and reoperations. Thirty-day mortality was absent in both groups.

Conclusion These results suggest that the introduction of the ERAS protocol in laparoscopic TME leads to a further reduction in length of hospital stay.
\end{abstract}

C. J. A. Huibers $\cdot$ M. A. J. de Roos $(\bowtie) \cdot$ K. H. Ong

Department of Surgery, Rivierenland Hospital,

Tiel, The Netherlands

e-mail: m.de.roos@zrt.nl
Keywords ERAS · Laparoscopic surgery · Rectal cancer . TME

\section{Introduction}

Laparoscopic surgery has several advantages over open surgery. These advantages are less postoperative pain, quicker return of bowel function, less postoperative morbidity and shorter duration of hospital stay $[1,2]$.

Laparoscopic TME is relatively new, but has proven to be a reliable and safe alternative for the conventional open TME. It seems that laparoscopic TME is the treatment of choice when there are no (relative) contraindications for laparoscopic surgery [3, 4].

The Enhanced Recovery after Surgery (ERAS) protocol, also known as 'fast-track' surgery, was originally developed to enhance postoperative recovery and thereby shorten hospital stay for patients undergoing elective colonic resection [2]. The first results of randomised trials are very promising, reporting postoperative hospital stay of $2-4$ days in favour of laparoscopic resections [5-7].

The key pathogenic factor in postoperative morbidity, when failures of surgical and anaesthetic techniques have been excluded, is the surgical stress response with subsequent increased demands on organ functions [8].

The body responds to surgical stress with profound changes in neural, endocrine and metabolic systems in addition to alterations in organ functions. Postoperatively, this may lead to complications such as myocardial infarction, delirium, pulmonary dysfunction, thromboembolism and infectious complications. Much effort was made to improve each of these specific outcome variables by unimodal therapeutic strategies [8]. The ERAS protocol, however, is a multimodal approach to all of these 'medical' complications. By reducing 
stress and pain in patients undergoing colorectal resections together with aggressive postoperative mobilisation and early oral feeding, the body's stress response and organ dysfunction are reduced to a minimum, thus facilitating early recovery and reducing postoperative morbidity and mortality [9].

With respect to postoperative recovery and the duration of postoperative hospital stay, both laparoscopic surgery and the ERAS protocol share the same objective. If both laparoscopic TME and ERAS are combined, can additional advantages be expected concerning postoperative recovery and the duration of hospital stay?

In order to investigate this question, a consecutive series of patients treated for rectal cancer were retrospectively divided in two groups. One group consisted of patients treated in the pre-ERAS era (the conventional care group), and the other group formed the ERAS group. Both groups were compared for length of postoperative hospital stay.

\section{Patients and methods}

All 124 patients diagnosed with rectal cancer in a single centre between January 2004 and July 2009 were analysed. Eightyfour patients underwent an elective laparoscopic TME. Eight patients were excluded due to conversion to open surgery. The remaining 76 patients were all included in this study.

The ERAS protocol for laparoscopic rectal surgery was introduced in this hospital in January 2007. Therefore, all patients operated between January 2007 and July 2009 formed the ERAS group $(N=43)$. The patients that underwent a rectal resection between January 2004 and January 2007 were assigned as a control group $(N=33)$.

All data were retrospectively collected from patient charts. Collected data included age, body mass index (BMI), smoking, hypertension, diabetes mellitus, the American Society of Anaesthesiologists' (ASA) classification, the type of procedure, operation time, estimated blood loss, tumour distance from the anal verge, preoperative treatment, type of anastomosis, creation of a temporarily diverting or permanent stoma, return of bowel function after surgery, postoperative complications, readmission and reoperation rate, 30-day mortality and the primary outcome: length of postoperative hospital stay (LOS). In addition, pathologic tumour characteristics were obtained.

Tumour distance in centimetres from the anal verge was measured using a rigid sigmoid scope. Preoperative treatment consisted either of a limited $(5 \times 5.0 \mathrm{~Gy})$ or extended $(25 \times 2.0 \mathrm{~Gy})$ radiotherapy $(\mathrm{RT})$ regimen or chemo radiation $(25 \times 2.0$ Gy + capecitabine $)$. All patients underwent either a laparoscopic low anterior resection (LAR) or a laparoscopic abdominal perineal resection (APR) according to the TME principle. In both groups the operations were performed by the same three surgeons, who were all well-trained in laparoscopic rectal surgery. The type of anastomosis was defined as end-to-end, end-to-side or side-to-side anastomosis. Patients either received a diverting loop ileostomy or, in the case of APR, a permanent colostomy. Return of bowel function was defined as the first reported stoma production. The postoperative complications considered were bleeding, wound infection, anastomotic leakage and intra-abdominal abscess. Anastomotic leakage was defined as clinical signs of peritonitis together with a visualized anastomotic defect on colonoscopy or colonic enema radiography, with or without an intraabdominal abscess. Intra-abdominal abscesses were radiographically detected pockets of fluid in a presacral cavity. The length of hospital stay was defined as the number of postoperative days in the hospital (day of surgery $=$ day 0 ). Readmission days were not added to this calculation. Readmissions and reoperations within 30 days after surgery were scored as either "yes" or "no". Readmissions and reoperations more than 30 days after discharge were not evaluated. Tumour characteristics comprising maximal tumour diameter on pathological examination, tumour differentiation grade, TNMclassification and radicality of the resection were obtained.

\section{The pre-ERAS protocol}

Patients were admitted the day before their operation. They received $4 \mathrm{~L}$ of oral bowel preparation (Klean-prep ${ }^{\circledR}$ Norgine B.V., Amsterdam, The Netherlands). A daily dose of $0.3 \mathrm{ml}$ Fraxiparine ${ }^{\circledR}$ was administered. Patients were allowed a normal diet until midnight.

In the operating room all patients received a urinary catheter. Thirty minutes before the first incision, cefazoline $(1,000 \mathrm{mg})$ and metronidazole $(500 \mathrm{mg})$ were given intravenously. Patients were operated under general anaesthesia. Nasogastric tubes were routinely used. In general, one drain was placed in the pelvis.

Postoperatively, oral intake was prohibited, and standard intravenous fluid was set at 2-2.5 L/24 h. Patients received 4,000 $\mathrm{mg}$ of paracetamol (in four separate doses of 1,000 mg). If necessary, diclofenac $150 \mathrm{mg}$ in three doses of $50 \mathrm{mg}$ and morphine substitutes were also given. Nasogastric tubes, drains and catheters were removed at the surgeon's decision. After removal of the nasogastric tube, patients were allowed clear fluids. Discharge was arranged when the following criteria were met: There are no remaining lines or catheters, solid food is tolerated, there has been passage of stool, pain is controlled using oral analgesics only and the patient is able to restart basic daily activities and self-care, or function at the preoperative level.

\section{The ERAS protocol}

Within the ERAS protocol perioperative care is standardised to promote early mobilisation and resumption of a normal 
diet as first described by H. Kehlet [5, 8, 10]. All patients that received the 'multimodal approach' (ERAS) agreed to an informed consent preoperatively in an outpatient setting. Patients were admitted the day before their operation to receive an epidural catheter, placed at a low level (Th 911, Table 1). Oral bowel preparation was omitted, but patients received two enemas on the evening before surgery.

Table 1 The ERAS protocol

The ERAS protocol

Day before surgery

- Normal diet until midnight

- Omission of oral bowel preparation

- 2 enemas in the evening

- An epidural catheter is placed at Th 9-11

- $0.3 \mathrm{ml}$ Fraxiparine, $4000 \mathrm{mg}$ paracetamol, $20 \mathrm{mg}$ pantoprazole (every day until discharge)

Day of surgery

- 1 enema in the morning

- 2 packages of carbohydrate drinks

- Oral intake until $2 \mathrm{~h}$ before surgery

- Target body temperature $\geq 36^{\circ} \mathrm{C}$

- Urinary catheter is placed

- Dexamethasone (5 $\mathrm{mg}$ ) and ondansetrone $(4 \mathrm{mg}$ ) preoperatively

- Cefalozine $(1000 \mathrm{mg})$ and metronidazole $(500 \mathrm{mg})$ i.v. $30 \mathrm{~min}$ before first incision

- Haloperidol (1.5 mg) intraoperatively

- Ventilation with $60-80 \%$ oxygen

- No nasogastric tubes

- All analgesics through the epidural catheter

- 1 intra-abdominal drain

- Oral fluid intake allowed and stimulated directly after surgery

- i.v. fluid restriction of $1 \mathrm{~L}$ per $24 \mathrm{~h}$

- Liquid diet in the evening

Postoperative day 1

- Disconnection of the intravenous catheter

- Start magnesium oxide

- Restart normal diet at noon

- 2-3 h of mobilisation

Postoperative day 2

- Diclofenac 50 mg, 3 times daily

- Removal of epidural catheter (when pain is controlled properly)

- Removal of urinary catheter and intra-abdominal drain

- Full mobilisation

Discharge criteria

- No remaining lines or catheters

- Toleration of solid food

- There has been passage of stool

- Pain can be controlled using only oral analgesics

- Ability to conduct self-care or function at preoperative level
Daily doses of $0.3 \mathrm{ml}$ Fraxiparine ${ }^{\circledR}, 4,000 \mathrm{mg}$ of paracetamol (in four separate doses of $1,000 \mathrm{mg}$ ) and $20 \mathrm{mg}$ of pantoprazole were administered, starting on the day before surgery and continued until discharge. Patients were allowed to maintain their normal diet until midnight. On the day of surgery patients received another enema and two packages of carbohydrate drinks. Patients were allowed oral intake until $2 \mathrm{~h}$ before surgery.

In the operating room body temperature was maintained using warm blankets. Target body temperature was $\geq 36^{\circ} \mathrm{C}$. All patients received a urinary catheter. Thirty minutes before the first incision, cefazoline $(1,000 \mathrm{mg})$ and metronidazole $(500 \mathrm{mg})$ were given intravenously. This was repeated every $3.5 \mathrm{~h}$. Five milligrams of dexamethasone and $4 \mathrm{mg}$ of ondansetrone were given preoperatively, and $1.5 \mathrm{mg}$ haloperidol was given intraoperatively. Patients were ventilated with 60 to $80 \%$ oxygen. Nasogastric tubes were not routinely used. All analgesics were given through the epidural catheter (levobupivacaine and sufentanil); the use of morphine and diclofenac was avoided. In general, one intraabdominal drain was left behind.

Postoperatively, oral fluid intake was directly encouraged, and intravenous fluid was restricted to $1 \mathrm{~L}$ per $24 \mathrm{~h}$. In the evening after surgery, a liquid diet was introduced. On the first day after surgery, if oral fluid intake was sufficient, the intravenous catheter was disconnected. Magnesium oxide was started, and the patient was allowed to restart a normal diet at noon. Patients were stimulated to mobilise $2-3 \mathrm{~h}$ on the first day.

On the second day after surgery, diclofenac was started in a dose of $50 \mathrm{mg}$, three times daily, and the epidural catheter was removed when pain was controlled properly (VAS $<4$ / 10). The urinary catheter and unproductive abdominal drains were removed, and patients were stimulated to fully mobilise.

Discharge was arranged when the following criteria were met: There are no remaining lines or catheters, solid food is tolerated, there has been passage of stool, pain is controlled using oral analgesics only and the patient is able to restart basic daily activities and self-care, or function at the preoperative level.

\section{Statistical analysis}

All collected data were entered in a database and analysed using SPSS Statistics 17.0 for Windows. To determine significant differences between the ERAS and control groups, statistical analysis was performed using a $\chi^{2}$ test. A $p$-value $<0.05$ was considered statistically significant.

Continuous data are expressed as median (range) or as mean $( \pm \mathrm{SD})$ and were analysed using Mann-Whitney $U$ test. BMI was converted to a categorical variable, representing 
certain risk groups. All categorical and dichotomous variables were analyzed using $\chi^{2}$ test.

Primary outcome was length of hospital stay. To determine independent outcome predictors, all possible predictors of the primary outcome were analysed in univariate and multivariate regression models. In the multivariate analysis, the backward regression method was used.

\section{Results}

In all patients in the ERAS group (43), an epidural catheter was successfully placed. In two (4.7\%) patients, the epidural catheter had to be removed before day 2 because of dislocation of the catheter. Twenty-one patients (49\%) could be disconnected from the intravenous catheter on day 1. In 37 $(86.0 \%)$ patients the epidural catheter could be removed on day 2 .Twenty-one patients $(49 \%)$ had their urinary catheter removed on day 2. In total, 16 (37\%) patients were managed exactly according to the ERAS protocol.

Both groups were compared for demographics and patient-related and tumour-related factors (Table 2). No significant differences were found between both groups, except for a history of diabetes. Nine patients in the ERAS group and one in the conventional care group had diabetes. Nearly all patients received preoperative treatment, consisting of a short scheme $(5 \times 5.0 \mathrm{~Gy})$ radiotherapy $(\mathrm{RT})$, long scheme $(25 \times 2.0 \mathrm{~Gy}) \mathrm{RT}$ or chemo radiation $(25 \times 2.0 \mathrm{~Gy}+$ capecitabine). Chemo radiation is a relatively new treatment regimen for patients with locally advanced rectal cancer. Therefore, this therapy was only observed in the ERAS group. All patients underwent either laparoscopic low anterior resection (LAR) or laparoscopic abdominal perineal resection (APR). There was no difference between both groups regarding the type of procedure.

Mean (total) operating time was $319 \pm 75 \mathrm{~min}$ in the ERAS group and $356 \pm 91 \mathrm{~min}$ in the conventional care group ( $p=0.068)$. Median blood loss was $300 \mathrm{ml}(0-2400)$ and $400 \mathrm{ml}(0-2400)$ respectively $(p=0.346)$. Operating time tended to be longer for LAR, and mean blood loss tended to be more for APR, but the differences did not reach statistical significance (Table 3).

There is a significant difference in length of postoperative hospital stay between both groups. The median postoperative hospital stay was 10 (4-74) days for patients receiving conventional care and 7 (2-83) days for patients that had followed the ERAS protocol ( $p=0.042)$. Furthermore, return of bowel function occurred significantly earlier in the ERAS group compared to the control group, with a median of 2 (1-7) and $3(1-14)$ days respectively $(p<0.001)$.

No significant differences were found when both groups were compared for postoperative complications, factors delaying early discharge, and readmission and reoperation
Table 2 Baseline characteristics

\begin{tabular}{|c|c|c|c|}
\hline & $\begin{array}{l}\text { No ERAS } \\
(N=33)\end{array}$ & $\begin{array}{l}\text { ERAS } \\
(N=43)\end{array}$ & $p$-Value* \\
\hline \multicolumn{4}{|l|}{ Age (years) } \\
\hline Median (range) & $64(27-88)$ & $66(36-79)$ & 0.156 \\
\hline Gender & & & 0.726 \\
\hline Male & $22(66.7)$ & $27(62.8)$ & \\
\hline Female & $11(33.3)$ & $16(37.2)$ & \\
\hline BMI & & & 0.456 \\
\hline$<25$ & $17(58.6)$ & $16(41)$ & \\
\hline $25-30$ & $7(24.1)$ & $13(33.3)$ & \\
\hline $30-35$ & $2(6.9)$ & $6(15.4)$ & \\
\hline$>35$ & $3(10.3)$ & $4(10.3)$ & \\
\hline Smoking $(N=63)$ & $8(24.2)$ & $10(23.3)$ & 0.872 \\
\hline Hypertension & $10(30.3)$ & $16(38.1)$ & 0.482 \\
\hline Diabetes & $1(3)$ & $9(21.4)$ & 0.020 \\
\hline ASA classification & & & 0.484 \\
\hline I & $9(27.3)$ & $18(41.9)$ & \\
\hline II & $17(51.5)$ & $15(34.9)$ & \\
\hline III & $6(18.2)$ & $9(20.9)$ & \\
\hline IV & $1(3)$ & $1(2.3)$ & \\
\hline UICC stage & & & 0.155 \\
\hline 0 & $1(3.0)$ & $1(2.3)$ & \\
\hline I & $12(36.4)$ & $11(25.6)$ & \\
\hline II & $13(39.4)$ & $11(25.6)$ & \\
\hline III & $7(21.2)$ & $20(46.5)$ & \\
\hline Preoperative treatment & & & $<0.001$ \\
\hline No preoperative treatment & $4(12.1)$ & $1(2.3)$ & \\
\hline RT short (5×5.0 Gy) & $19(57.6)$ & $23(53.5)$ & \\
\hline RT long $(25 \times 2.0$ Gy $)$ & $10(30.3)$ & $3(7)$ & \\
\hline $\begin{array}{l}\text { Chemo radiation } \\
(25 \times 2.0 \text { Gy }+ \text { capecitabine })\end{array}$ & 0 & $16(37.2)$ & \\
\hline $\begin{array}{l}\text { Tumour distance from anal } \\
\text { verge }(\mathrm{cm})\end{array}$ & & & 0.635 \\
\hline $0-4$ & $12(36.4)$ & $18(41.9)$ & \\
\hline $5-8$ & $14(42.4)$ & $17(39.5)$ & \\
\hline$>8$ & $7(21.2)$ & $8(18.6)$ & \\
\hline Procedure & & & 0.228 \\
\hline $\begin{array}{l}\text { Lap. low anterior resection } \\
\text { (LAR) }\end{array}$ & $25(75.8)$ & $27(62.8)$ & \\
\hline $\begin{array}{l}\text { Lap. abdominal perineal } \\
\text { resection (APR) }\end{array}$ & $8(24.2)$ & $16(37.2)$ & \\
\hline
\end{tabular}

Values in parentheses are percentages unless indicated otherwise $B M I$ body mass index, ASA American Society of Anaesthesiology, $U I C C$ Union for International Cancer Control, $R T$ radiotherapy

$* p$-Value calculated by $\chi^{2}$ test

rates within 30 days after surgery. In both groups 30-day mortality was absent.

Haemorrhage occurred in one patient in the ERAS group ( $p=0.38)$; this patient needed reoperation. Four $(9.3 \%)$ ERAS patients developed a wound infection and two $(6.1 \%)$ patients 
Table 3 Results

Values in parentheses are percentages unless indicated otherwise

$L A R$ low anterior resection, $A P R$ abdominal perineal resection

* $p$-Value calculated using Mann-Whitney $U$ test;

$* * p$-value calculated using

$\chi^{2}$ test

${ }^{\mathrm{a}}$ Cardiovascular events, pneumonia and urinary tract infections

\begin{tabular}{|c|c|c|c|}
\hline & No ERAS $(N=33)$ & ERAS $(N=43)$ & $p$-Value \\
\hline $\begin{array}{l}\text { Operating time }(\min )(\text { mean } \pm \text { SD) } \\
\text { LAR }\end{array}$ & $\begin{array}{l}356 \pm 91 \\
368 \pm 96\end{array}$ & $\begin{array}{l}319 \pm 75 \\
323 \pm 76\end{array}$ & $0.068^{*}$ \\
\hline APR & $318 \pm 64$ & $313 \pm 74$ & \\
\hline $\begin{array}{l}\text { Total blood loss (ml) (median (range)) } \\
\text { LAR }\end{array}$ & $\begin{array}{l}400(0-2400) \\
400(0-2400)\end{array}$ & $\begin{array}{l}300(0-2400) \\
250(0-1700)\end{array}$ & $0.346^{*}$ \\
\hline APR & $600(0-1100)$ & $425(0-2400)$ & \\
\hline Length of stay (days) & & & $0.042 *$ \\
\hline Median (range) & $10(4-74)$ & $7(2-83)$ & \\
\hline Return of bowel function (days) & & & $<0.001^{*}$ \\
\hline Median (range) & $3(1-14)$ & $2(1-7)$ & \\
\hline \multicolumn{4}{|l|}{ Postoperative complications } \\
\hline Bleeding & 0 & $1(2.3)$ & $0.378 * *$ \\
\hline Wound infection & $2(6.1)$ & $4(9.3)$ & $0.603 * *$ \\
\hline Anastomotic leakage & $4(16.0)$ & $5(18.5)$ & $0.947 * *$ \\
\hline Intra-abdominal abscess & $3(9.1)$ & $7(16.3)$ & $0.358 * *$ \\
\hline Factors complicating early discharge & & & $0.216^{* *}$ \\
\hline No complications & $12(36.4)$ & $19(44.2)$ & \\
\hline Gastro-intestinal complications & $10(30.3)$ & $5(11.6)$ & \\
\hline Urological complications & $6(18.2)$ & $13(30.2)$ & \\
\hline Other $^{\mathrm{a}}$ & $5(15.2)$ & $6(13.9)$ & \\
\hline Readmission ( $<30$ days after surgery) & $6(18.2)$ & $5(11.6)$ & $0.421 * *$ \\
\hline Reoperation ( $<30$ days after surgery) & $5(15.2)$ & $7(16.3)$ & $0.894 * *$ \\
\hline Mortality (<30 days after surgery) & 0 & 0 & \\
\hline
\end{tabular}

in the conventional care group $(p=0.60)$. Anastomotic leakage occurred in five $(11.6 \%)$ and four $(12.1 \%)$ patients respectively $(p=0.95)$. Intra-abdominal abscesses were reported in seven $(16.3 \%)$ and three $(9.1 \%)$ cases respectively $(p=0.36)$. Furthermore, factors delaying early discharge were reported and divided into gastro-intestinal factors (ileus, gastric paresis), urological factors (bladder retention, prolonged requirement of urinary catheter) and other factors (cardiovascular events, pneumonia and urinary tract infections). No significant differences were found between both groups regarding these factors.

LOS was converted to a dichotomous variable, in order to be able to perform logistic regression. The median hospital stay of all patients was 8 days. Therefore, LOS of 1-7 days and $\geq 8$ days were used as cutoff points. All variables with $p$ values less than 0.2 in the univariate analysis were entered in the multivariate regression model. After univariate analysis, four possible predictors for length of hospital stay were identified, including: age at time of operation, ASA classification, diabetes and the ERAS protocol (Table 4). Preoperative treatment was added to the multiple regression analysis due to the significant differences between the ERAS and control groups at baseline. After multivariate analysis, both diabetes (OR $0.12,95 \%$ CI $0.02-0.66, p=$ 0.015 ) and the ERAS protocol (OR 5.14, 95\% CI 1.80$14.65, p=0.002$ ) were independent predictors of the outcome, a hospital stay of 1-7 days.

\section{Discussion}

In this study the authors found that since the introduction of the ERAS protocol in laparoscopic TME for rectal cancer, postoperative hospital stay was significantly reduced and bowel function was restored earlier compared to laparoscopic TME and conventional care. Although the results seem very promising, the authors realize that this is a retrospective study from which no definitive conclusions can be drawn.

Table 4 Univariate analysis investigating the effect on LOS (Univariate results of variables added to the multivariate analysis)

\begin{tabular}{llll}
\hline Variable & OR & $95 \%$ CI & $p$-Value \\
\hline Age & 1.04 & $0.99-1.08$ & 0.055 \\
ASA I & 1 & Reference & 0.096 \\
ASA II & 0.39 & $0.13-1.13$ & 0.081 \\
ASA III & 0.18 & $0.05-0.74$ & 0.017 \\
ERAS & 3.52 & $1.35-9.20$ & 0.010 \\
Diabetes & 0.24 & $0.48-1.23$ & 0.087 \\
Chemo radiation & 1 & Reference & 0.235 \\
Short $(5 \times 5.0 \mathrm{~Gy})$ & 0.31 & $0.09-1.05$ & 0.060 \\
Long $(25 \times 2.0 \mathrm{~Gy})$ & 0.28 & $0.06-1.32$ & 0.109 \\
\hline
\end{tabular}

$O R$ odds ratio, $C I$ confidence interval, $A S A$ American Society of Anaesthesiology 
To our knowledge no comparative studies on ERAS and conventional care in laparoscopic rectal surgery have been published so far. One recent study reported the results of laparoscopic rectal resections combined with fast-track surgery [11]. In that study $(N=37)$ the authors found a mean hospital stay of 3 days (range 1-8). This is an excellent result which has not been published before. However, there were only $46 \%$ of the patients in the study that had a rectal carcinoma, and all patients underwent a laparoscopic LAR. In $19 \%$ a diverting loop ileostomy was performed. Mean hospital stay in that group was 4 days (range 2-7). The 30day readmission rate was $8 \%$ and was not elevated by early discharge. No anastomotic leakage occurred.

Similar results regarding LOS have been described for laparoscopic colonic resections, but rectal resections have been associated with median hospital stays of 12 or 13 days. The introduction of the laparoscopic technique for TME has led to a reduction in LOS, with a median hospital stay of approximately 10 days [12-14]. This corresponds with the results in the control group in our study (median hospital stay of 10 days).

In our study, nearly all patients (98\%) that underwent a LAR with an anastomosis received a temporary diverting stoma. Recently, a randomised multicenter trial, a multicenter analysis and meta-analysis have been published on this subject, reporting a significant decline in symptomatic anastomotic leakage after low anterior resections when a diverting stoma was performed [15-18]. However, many recent studies on laparoscopic low anterior resections showed a temporary diversion rate as low as $19-26 \%$, suggesting this assumption is not widely accepted $[11,19,20]$. The symptomatic anastomotic leakage rate among all low anterior resections performed in our study was $17.3 \%$, which is relatively high compared to other studies including patients with a diverting stoma $(7.8 \%$ in a multicenter analysis) [16]. This could partly be due to the preoperative treatment. Seven of these nine patients received short scheme RT followed by a LAR. The other two patients received a long scheme RT. Preoperative RT has been associated with higher anastomotic leakage rates, compared with no preoperative treatment [21-23]. Furthermore, in our study LAR was also performed in cases with very low tumours located at $2-4 \mathrm{~cm}$ from the anal verge. Low anastomoses have also been associated with higher rates of anastomotic leakages [22, 23].

In addition, a stoma requires extra postoperative care and can be a source of postoperative complications. Furthermore, patients could only be discharged when stoma care was sufficient. Therefore, the presence of a stoma could be a factor delaying early discharge. There was no difference in diversion rate between ERAS and control groups. Nevertheless, the overall hospital stay might have been shorter and better comparable with other study results if a diverting stoma was not routinely performed.

Both groups were comparable for demographics and patient-related factors, except for the presence of diabetes, which was more likely to occur in the ERAS group $(N=9)$ compared to the control group $(N=1)$. This will not bias our results in favour of our hypothesis because diabetes has been associated with delayed wound healing and impaired anastomotic strength. These factors are more likely to complicate and delay recovery than accelerate it [24, 25]. Additionally, the multivariate regression analysis supported this assumption. As mentioned earlier, the preoperative therapy with chemo radiation was only used in the ERAS group. Long scheme RT was more frequent within the control group. Patients treated with chemo radiation preoperatively are more prone to develop postoperative complications such as wound healing problems and anastomotic dehiscence, due to elevated metalloproteinase activity $[26,27]$. The higher incidence of chemo radiation in the ERAS group would therefore be more of a disadvantage than an advantage with respect to postoperative recovery and length of hospital stay, possibly even underestimating the results presented in this study. On the other hand, no higher anastomotic leakage rate was found in the chemo radiation group.

Another possible confounder of the study could be the learning curve for a surgeon to become an expert in laparoscopic colorectal surgery. The laparoscopic technique for TME was introduced in our institution in 2003. In order to rule out factors that could be associated with the learning curve of the surgeons, we excluded all patients who received a laparoscopic rectal resection in the first year after the introduction of this technique. However, the learning curve for laparoscopic left sided colorectal resections is long (up to 62 cases) [28]. The surgeons in our institution had done approximately $25-30$ cases per surgeon in the first year. The mean operating time, a factor that is negatively correlated with the learning curve, tended to be longer in the control group, but this difference did not reach statistical significance $(p=0.068)$. The conversion rate, also associated with a learning curve, was $6.5 \%$ in the ERAS group and $13.2 \%$ in the control group $(p=0.30)$. Although both variables that indicated the presence of a learning curve did not differ significantly between the ERAS and control groups, we cannot safely exclude these factors as possible confounders in our results, based on our relatively small study sample.

After reviewing factors that could possibly delay discharge, we found a trend towards more urological complications in the ERAS group. Many patients required a urinary catheter longer than 2 days postoperatively (according to the protocol), mostly because of persistent urinary bladder retention. Twelve patients within this group even left the hospital with their urinary catheter still in place. We could not find a reasonable explanation for the difference between both groups regarding the urological complications. There could be a correlation with the preoperative treatment (chemo radiation), possibly leading to more nerve damage, but there are no studies confirming this hypothesis. Another explanation could be a better 
registration of urological complications in the ERAS group by the nursing and medical staff, leading to possible bias.

\section{Recommendations}

Although this study shows a significant reduction in hospital stay after the introduction of the ERAS protocol in laparoscopic TME for rectal cancer, no definitive conclusions can be drawn. The results suggest this decline in postoperative hospital stay is directly related to the ERAS protocol. However, based on our study design, we cannot confirm ERAS to be the causal factor.

In the future, a randomised controlled clinical trial is required to validate the results we presented here.

Disclosures CJA Huibers, MAJ de Roos and KH Ong have no conflicts of interest or financial ties to disclose.

Open Access This article is distributed under the terms of the Creative Commons Attribution Noncommercial License which permits any noncommercial use, distribution, and reproduction in any medium, provided the original author(s) and source are credited.

\section{References}

1. Milsom JW, de Oliveira O Jr, Trencheva KI, Pandey S, Lee SW, Sonoda T (2009) Long-term outcomes of patients undergoing curative laparoscopic surgery for mid and low rectal cancer. Dis Colon Rectum 52(7):1215-1222

2. Milsom JW, Bohm B, Hammerhofer KA, Fazio V, Steiger E, Elson P (1998) A prospective, randomized trial comparing laparoscopic versus conventional techniques in colorectal cancer surgery: a preliminary report. J Am Coll Surg 187(1):46-54

3. Breukink S, Pierie J, Wiggers T (2006) Laparoscopic versus open total mesorectal excision for rectal cancer. Cochrane Database Syst Rev (4):CD005200

4. Laurent C, Leblanc F, Wutrich P, Scheffler M, Rullier E (2009) Laparoscopic versus open surgery for rectal cancer: long-term oncologic results. Ann Surg 250(1):54-61

5. Kehlet H, Wilmore DW (2008) Evidence-based surgical care and the evolution of fast-track surgery. Ann Surg 248(2):189-198

6. Wind J, Polle SW, Fung Kon Jin PH, Dejong CH, von Meyenfeldt MF, Ubbink DT, Gouma DJ, Bemelman WA (2006) Systematic review of enhanced recovery programmes in colonic surgery. Br J Surg 93(7):800-809

7. Khoo CK, Vickery CJ, Forsyth N, Vinall NS, Eyre-Brook IA (2007) A prospective randomized controlled trial of multimodal perioperative management protocol in patients undergoing elective colorectal resection for cancer. Ann Surg 245(6):867-872

8. Kehlet H (1997) Multimodal approach to control postoperative pathophysiology and rehabilitation. Br J Anaesth 78(5):606-617

9. Spanjersberg WR, Reurings J, van Laarhoven CHJM (2009) Fast track surgery versus conventional recovery strategies for colorectal surgery. Cochrane Database Syst Rev (1):CD007635

10. Kehlet H (2009) Multimodal approach to postoperative recovery. Curr Opin Crit Care 15(4):355-358

11. Lindsetmo RO, Champagne B, Delaney CP (2009) Laparoscopic rectal resections and fast-track surgery: what can be expected? Am J Surg 197(3):408-412
12. Veenhof AA, Engel AF, Craanen ME, Meijer S, de Lange-de Klerk ES, van der Peet DL, Meijerink WJ, Cuesta MA (2007) Laparoscopic versus open total mesorectal excision: a comparative study on shortterm outcomes. A single-institution experience regarding anterior resections and abdominoperineal resections. Dig Surg 24(5):367-374

13. Hasegawa H, Ishii Y, Nishibori H, Endo T, Watanabe M, Kitajima M (2007) Short- and midterm outcomes of laparoscopic surgery compared for 131 patients with rectal and rectosigmoid cancer. Surg Endosc 21(6):920-924

14. Staudacher C, Vignali A, Saverio DP, Elena O, Andrea T (2007) Laparoscopic vs. open total mesorectal excision in unselected patients with rectal cancer: impact on early outcome. Dis Colon Rectum 50(9):1324-1331

15. Matthiessen P, Hallbook O, Rutegard J, Simert G, Sjodahl R (2007) Defunctioning stoma reduces symptomatic anastomotic leakage after low anterior resection of the rectum for cancer: a randomized multicenter trial. Ann Surg 246(2):207-214

16. den Dulk M, Marijnen CA, Collette L, Putter H, Pahlman L, Folkesson J, Bosset JF, Rodel C, Bujko K, van de Velde CJ (2009) Multicentre analysis of oncological and survival outcomes following anastomotic leakage after rectal cancer surgery. Br J Surg 96(9):1066-1075

17. Tan WS, Tang CL, Shi L, Eu KW (2009) Meta-analysis of defunctioning stomas in low anterior resection for rectal cancer. Br J Surg 96(5):462-472

18. Huser N, Michalski CW, Erkan M, Schuster T, Rosenberg R, Kleeff J, Friess H (2008) Systematic review and meta-analysis of the role of defunctioning stoma in low rectal cancer surgery. Ann Surg 248(1):52-60

19. Akiyoshi T, Kuroyanagi H, Oya M, Konishi T, Fukuda M, Fujimoto Y, Ueno M, Yamaguchi T (2009) Short-term outcomes of laparoscopic rectal surgery for primary rectal cancer in elderly patients: is it safe and beneficial? J Gastrointest Surg 13(9):1614-1618

20. Kuroyanagi H, Akiyoshi T, Oya M, Fujimoto Y, Ueno M, Yamaguchi T, Muto T (2009) Laparoscopic-assisted anterior resection with double-stapling technique anastomosis: safe and feasible for lower rectal cancer? Surg Endosc 23(10):2197-2202

21. Doeksen A, Tanis PJ, Vrouenraets BC, Gooszen JA, van Lanschot JJ, van Tets WF (2007) Outcome of rectal cancer surgery after the introduction of preoperative radiotherapy in a low-volume hospital. J Gastrointest Cancer 38(2-4):63-70

22. Matthiessen P, Hallbook O, Andersson M, Rutegard J, Sjodahl R (2004) Risk factors for anastomotic leakage after anterior resection of the rectum. Colorectal Dis 6(6):462-469

23. Taflampas P, Christodoulakis M, Tsiftsis DD (2009) Anastomotic leakage after low anterior resection for rectal cancer: facts, obscurity, and fiction. Surg Today 39(3):183-188

24. Verhofstad MH, Hendriks T (1994) Diabetes impairs the development of early strength, but not the accumulation of collagen, during intestinal anastomotic healing in the rat. Br J Surg 81 (7): $1040-1045$

25. Verhofstad MH, Lomme RM, de Man BM, Hendriks T (2002) Intestinal anastomoses from diabetic rats contain supranormal levels of gelatinase activity. Dis Colon Rectum 45(4):554-561

26. Rieff EA, Hendriks T, Rutten HJ, Nieuwenhuijzen GA, Gosens MJ, van den Brule AJ, Nienhuijs SW, de Hingh IH (2009) Neoadjuvant radiochemotherapy increases matrix metalloproteinase activity in healthy tissue in esophageal cancer patients. Ann Surg Oncol 16(5):1384-1389

27. Stumpf M, Klinge U, Wilms A, Zabrocki R, Rosch R, Junge K, Krones C, Schumpelick V (2005) Changes of the extracellular matrix as a risk factor for anastomotic leakage after large bowel surgery. Surgery 137(2):229-234

28. Tekkis PP, Senagore AJ, Delaney CP, Fazio VW (2005) Evaluation of the learning curve in laparoscopic colorectal surgery: comparison of right-sided and left-sided resections. Ann Surg 242(1):83-91 